\title{
Generation from continuous waves of frequency combs with large overall bandwidth and tunable central wavelength
}

\author{
B. Barviau, C. Finot, J. Fatome, G. Millot
}

We experimentally show that the combined effects of Raman soliton selffrequency shift with either compression of a sinusoidal beating or modulation instability lead to the generation of frequency combs with a spectral width higher than $100 \mathrm{~nm}$ and a central frequency shifted towards higher wavelengths.

Introduction: Generation of new optical frequencies in the 1.6-1.7 $\mu \mathrm{m}$ range is of high interest for a large variety of applications. In this context, starting from erbium-fibre based devices emitting in the $1.5 \mu \mathrm{m}$ range, several techniques based on nonlinear effects in optical fibres could be used, such as wavelength conversion through four-wave mixing [1], generation of cascaded Raman Stokes waves [2] or spectral slicing of supercontinuums [3]. However, those various approaches can suffer from serious drawbacks. Indeed, in the first case, the pump energy is equally spread between lower and higher wavelengths, thus leading to non-optimal efficiency for the 1.6-1.7 $\mu \mathrm{m}$ range. For Raman emission, a restricted number of frequencies is allowed due to the large and fixed spectral spacing resulting from the Raman gain of the propagation medium, e.g. silica or gas filling a hollow-core photonic bandgap fibre. On the other hand when using a supercontinuum, initial energy is 
continuously spread out over a broad spectral range, thus leading to a relatively low spectral power density.

In this paper, we experimentally evidence an alternative approach exploiting the soliton self-frequency shift (SSFS) in order to tune the central frequency of two continuous or quasi-continuous waves [4]. We will first expose the principle of the method before detailing the experimental device and the results obtained by two different alternatives.

Method principle: An attractive and flexible solution for shifting the wavelength of an optical wave is the use of intrapulse Raman scattering under which the central frequency of a wave is progressively shifted towards lower frequencies [5]. However, as this process is only efficient for ultrashort pulses, it does not seem a priori suitable when continuous or quasi-continuous (nanosecond pulses) waves are considered. So, the issue was to find an easy way to convert two continuous waves into a train of sub-picosecond pulses. Two approaches are then feasible. The first one is based on the compression of a sinusoidal beat signal into a train of Gaussian transform-limited ultrashort pulses [6]. For this technique, energy is initially equally divided between two wavelengths and harmonics develop through a multiple four-wave mixing process. In the second approach, energy is mainly concentrated into the pump wavelength, while a seed signal stimulates the generation of harmonics via induced modulation instability [7]. Once the train of ultrashort pulses is achieved, then it will be subject to the SSFS process. 
In both approaches, we will generate at the fibre output a frequency comb with a central wavelength shifted toward higher wavelengths. The frequency spacing between the harmonics can be tuned by simply changing the frequency separation between the two initial continuous waves. It should be noted that the conversion of quasi-continuous waves into an ultrashort pulse train and the SSFS effect proceed in a single anomalous dispersive optical fibre.

Experimental set-up: The general idea to combine modulation instability and SSFS was numerically suggested in 1990 by Mamyshev et al. [4] with the aim of filtering pedestals of ultrashort pulses. However, to date, no experimental implementation had still been carried out. We detail here the two experimental set-ups that we have implemented. The first approach (see Fig. 1) is based on the sinusoidal beat signal created by two continuous laser diodes spaced by $9 \mathrm{~nm}$ (external cavity lasers, ECL). An acousto-optic modulator makes it possible to slice long pulses (350 ns) which are then amplified by an erbium doped fibre amplifier (EDFA) with an average power of $1 \mathrm{~W}$. A phase modulator is used in order to avoid deleterious effects of Brillouin backscattering [6]. The continuous waves are then injected into a $900 \mathrm{~m}$ highly non-linear fibre (HNLF - nonlinearity $10 \mathrm{~W}^{-1} \cdot \mathrm{km}^{-1}$ ) with low anomalous dispersion $\left(0.7\right.$ ps. $\left.\mathrm{nm}^{-1} \cdot \mathrm{km}^{-1}\right)$, reduced dispersion slope of $0.0072 \mathrm{ps} . \mathrm{nm}^{-2} \cdot \mathrm{km}^{-1}$ and attenuation of $0.7 \mathrm{~dB} \cdot \mathrm{km}^{-1}$. Our second approach (see Fig. 2) relies on the 
use of 10 ns flat top pump pulses delivered by an erbium doped fibre laser. The pulse profile is supergaussian (Fig. 2b) and has thus the advantage of not presenting any notable rebound [8]. A peak-power of $17 \mathrm{~W}$ enables us to use a shorter segment of dispersion-flattened HNLF (90 m - same parameters as the fibre used in the first approach) as well as a seed pulse shifted by $25.5 \mathrm{~nm}$. Let us note that for both approaches, in order to optimize the efficiency of the temporal compression of the initial modulation, we have used linearly polarised sources and carefully aligned the polarisations at the HNLF input.

Results: The experimental results are presented in Fig. 3. In the case of the sinusoidal beat compression, 24 harmonics are generated between 1500 and $1710 \mathrm{~nm}$, regularly spaced by $9 \mathrm{~nm}$ (Fig. 3a). In the approach based on induced modulation instability, 11 harmonics are obtained between 1450 and $1750 \mathrm{~nm}$, regularly spaced by $25.5 \mathrm{~nm}$ (Fig. 3b). The signal-to-noise ratio measured with an optical spectrum analyser is higher than $17 \mathrm{~dB}$. Thanks to the large spectral period of the comb, a single harmonic could be easily extracted with a band-pass filter in order to generate a frequency-shifted continuous (or quasi-continuous) wave. In both methods, the spectrum is shifted via intrapulse Raman effect towards higher wavelengths (dominating wavelength of $1689 \mathrm{~nm}$ and $1635 \mathrm{~nm}$ for the first and second method, respectively). The central wavelengths of the combs can be easily tuned by adjusting both initial frequency separation and powers launched into the HNLF. 
Numerical integration of the generalized non-linear Schrödinger equation which governs the wave propagation has highlighted that the major effects limiting the performances achievable by both techniques are the third order dispersion and the initial signal to noise ratio. Moreover, building up of Raman amplified spontaneous emission can also partly explain the degradation of the output optical signal to noise ratio.

Conclusion: We have experimentally studied the combined effects of the Raman soliton self-frequency shift with either beat signal compression or induced modulation instability. Two fully-fibered set-ups, relying exclusively on commercially available devices, have enabled us to generate frequency combs with a spectral width higher than $100 \mathrm{~nm}$ and a central frequency shifted towards higher wavelengths. The use of an additional contrapropagative Raman pumping would make it possible to extend the observed spectral shift [9].

\section{References}

1. NOWAK G. A., KAO, Y. H., XIAO, T. J., ISLAM, M. N., and NOLAN, D. A.: "Low-power high-efficiency wavelength conversion based on modulational instability in high-nonlinearity fiber," Opt. Lett., 1998, 23, pp. 936-938 
2. BENABID F., KNIGHT, J. C., and RUSSELL, P. S.: "Stimulated Raman scattering in hydrogen-filled hollow-core photonic crystal fiber," Science, 2002, 298, pp. 399-402

3. ABEELUCK A. K., and HEADLEY, C.: "Continuous-wave pumping in the anomalous- and normal-dispersion regimes of nonlinear fibers for supercontinuum generation," Opt. Lett., 2005, 30, pp. 61-63

4. MAMYSHEV P. V., CHERNIKOV, S. V., DIANOV, E. M., and PROKHOROV, A. M.: "Generation of a high-repetition-rate train of praticaly noninteractiong solitons by using the induced modulational instability and Raman self-scattering effects.," Opt. Lett., 1990, 15, pp. 1365-1367

5. GORDON J. P.: "Theory of the soliton self-frequency shift," Opt. Lett., 1986, 11, pp. 662-664

6. FATOME J., PITOIS, S., and MILLOT, G.: "20-GHz-to-1-THz repetition rate pulse sources based on multiple four-wave mixing in optical fibers," IEEE J. Quantum Electron., 2006, 42, pp. 1038-1046

7. TAI K., HASEGAWA, A., and TOMITA, A.: "Observation of modulational instability in optical fibers," Phys. Rev. Lett., 1986, 56, pp. 135-138

8. KUZIN E. A., MENDOZA-VAZQUEZ, S., GUTIERREZ, J., IBARRAESCAMILLA, B., HAUS, J. W., and ROJA-LAGUNA, R.: "Intra-pulse Raman frequency shift versus conventional Stokes generation of diode laser pulses in optical fibers," Opt. Express, 2005, 13, pp. 3388-3396

9. CHESNUT D. A., and TAYLOR, J. R.: "Soliton self-frequency shift in highly nonlinear fiber with extension by external Raman pumping," Opt. Lett., 2003, 28, pp. 2512-2514

\section{Authors' affiliations:}

B. Barviau, C. Finot, J. Fatome and G. Millot * (Institut Carnot de Bourgogne, UMR 5209 CNRS-Université de Bourgogne, 9 Av. A. Savary, BP 47 870, F21078 DIJON Cedex, FRANCE)

* e-mail adress of the corresponding author : gmillot@u-bourgogne.fr 


\section{Figure captions:}

Fig. 1 Method based on the non-linear compression of a sinusoidal beating

(a) Experimental set-up.

(b) Autocorrelation signal of the initial beating.

(c) Initial spectrum.

Fig. 2 Method relying on the induced modulation instability.

(a) Experimental set-up.

(b) Intensity profile of the ns initial pulse recorded on a high speed oscilloscope.

(c) Initial spectrum.

Fig. 3 Experimental spectrum obtained after propagation in HNLF

(a) Method based on the non-linear compression of a sinusoidal beating.

(b) Method relying on the induced modulation instability. 
Figure 1
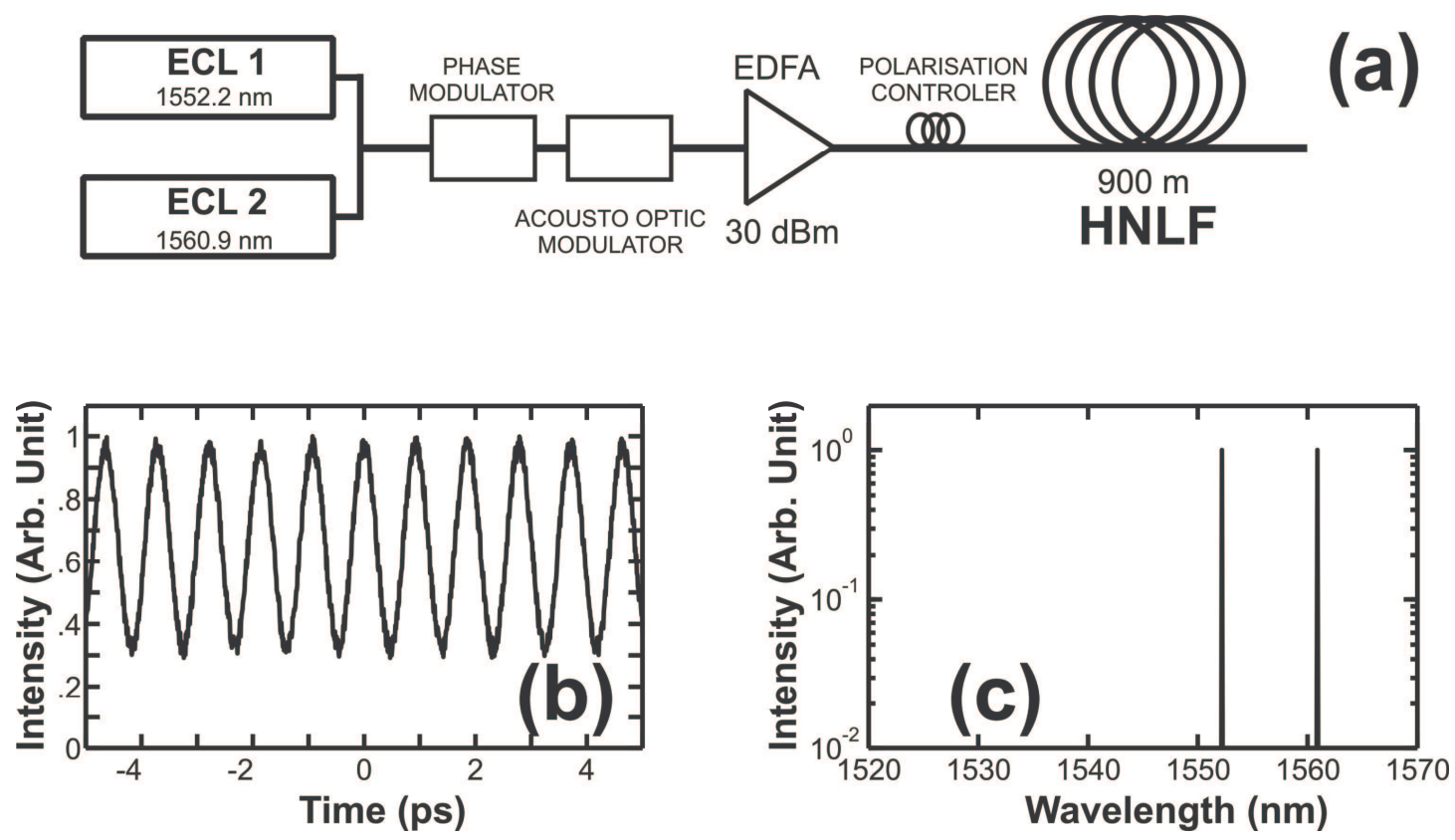
Figure 2
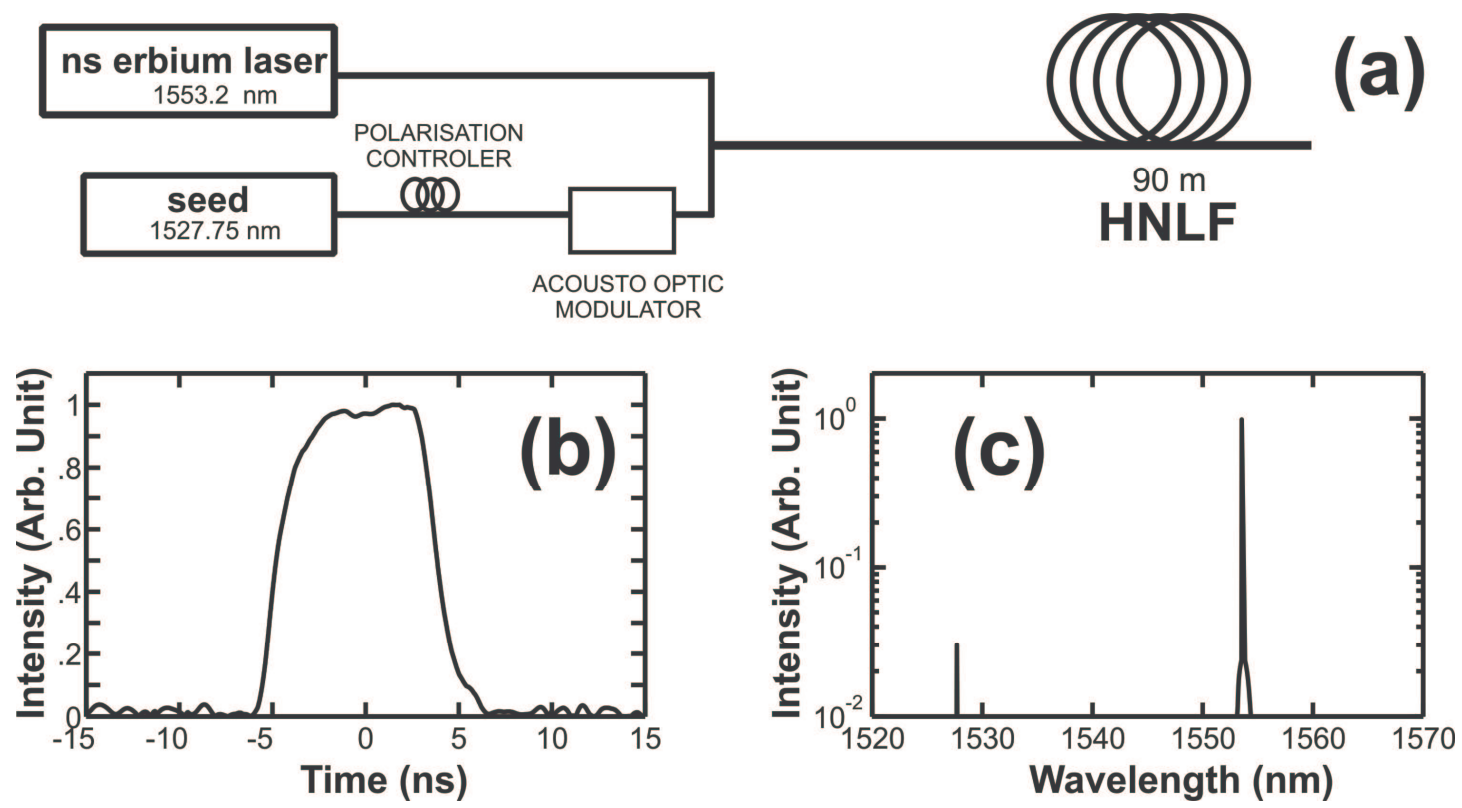
Figure 3

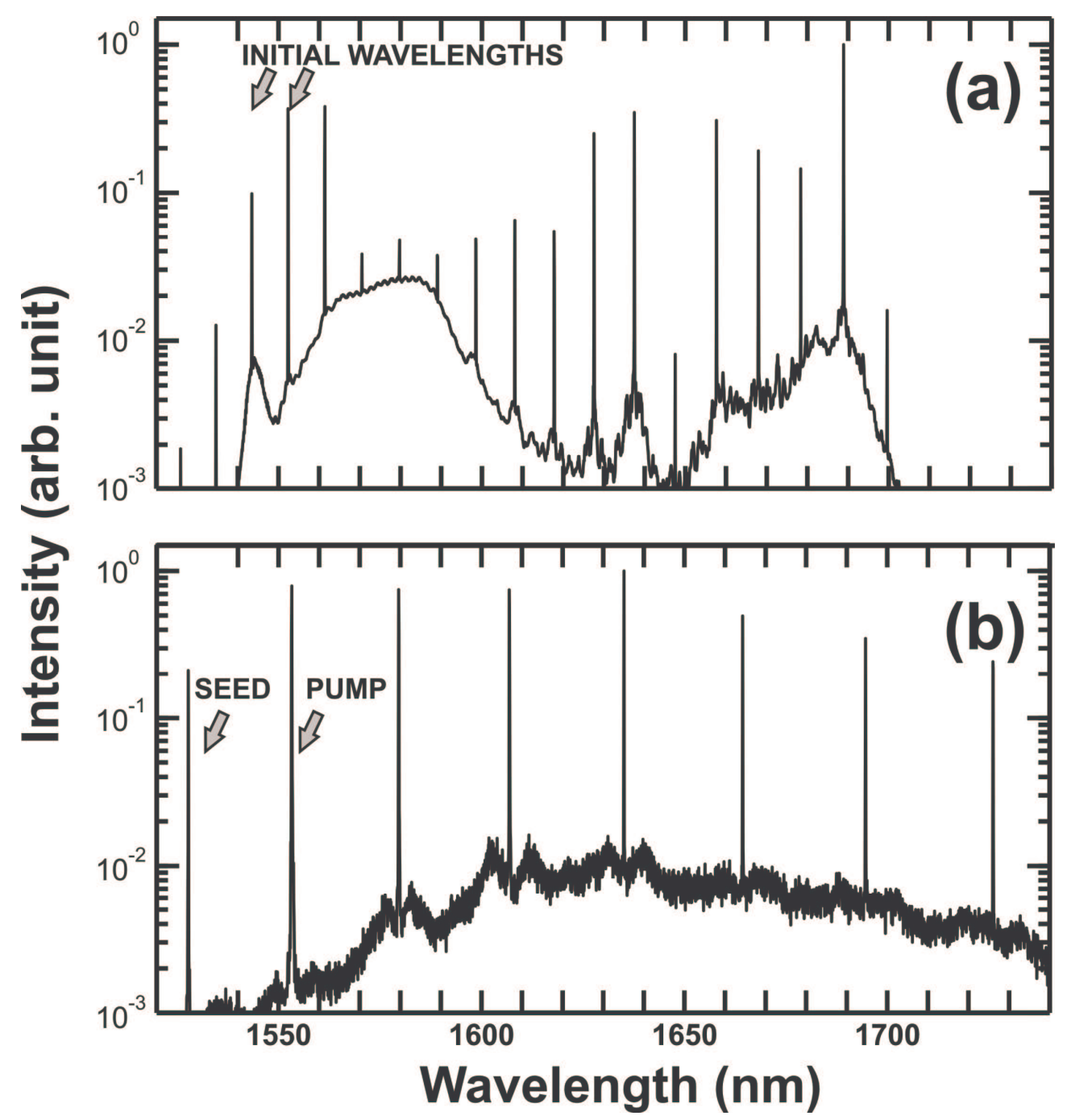

